\title{
ShiverPad: A Device Capable of Controlling Shear Force on a Bare Finger
}

\author{
Erik C. Chubb* \\ J. Edward Colgate ${ }^{\dagger}$ \\ Northwestern University \\ 2145 Sheridan Rd \\ Evanston IL 60208, USA
}

Michael A. Peshkin

\begin{abstract}
We discuss the theory, design, and performance of a flat-panel tactile display capable of controlling shear force on a finger. In previous research a $\mathrm{TPaD}$ variable friction device was presented. It modulates friction by using vertical ultrasonic vibrations to form a squeeze film of air between the finger and the horizontal glass surface. In this research, a TPaD is oscillated in the horizontal plane at $20-100 \mathrm{~Hz}$ while alternating between low and high friction at the same frequency. As the plate moves in one direction, the squeeze film is turned on and friction is reduced. As the plate moves in the opposite direction, the squeeze film is turned off and the friction increases. The net time-averaged force is non-zero and can be used as a source of shear force to a finger in contact with the surface. We demonstrate the implementation of line sources and sinks using this new technology.
\end{abstract}

Index Terms: H.5.2 [User Interfaces]: Haptic I/O; []

\section{INTRODUCTION}

There are several designs for tactile displays capable of applying shear force to a finger tip. The STReSS tactile display developed by Pasquero and Hayward [4] uses a $10 \times 10$ array of piezoelectric actuated contactors to create compressive and tensile strains in the skin of the fingertip. One advantage of an array of individually controlled contactors is that it can apply tensile and compressive stresses to different areas of the fingertip simultaneously. Levesque and Hayward's research [8] indicates that the fingertip experiences such stress distribution when it encounters edges or small bumps. Additionally, these devices can be used with a bare finger and do not require finger motion to produce force.

A second category of tactile displays relies on the principle that lateral forces can be used to create the illusion of texture and surface features. This idea was originated by Minsky [3] and furthered by Robles-De-La-Torres and Hayward [5], both of whom worked with kinesthetic displays. It has served as inspiration, however, for a number of flat panel tactile displays. For example, Yamamoto et al. [11] created a display that uses electrostatics to control frictional forces on the fingertip. The user rests his finger on a thin-film slider (or thimble) and the frictional forces between the slider and the substrate are controlled. One advantage of this device is that it can be made visually transparent and implemented on top of a visual display. Takasaki et al. [7] created a transparent device with a similar slider-based interface, except that friction modulation is achieved through surface acoustic waves. This $15 \mathrm{MHz}$ vibration creates a vertical motion on the order of $10 \mathrm{~nm}$ that results in periodic contact with the hard spheres on the underside of the slider. The periodic contact reduces the perceived friction.

\footnotetext{
*e-mail: erikchubb@u.northwestern.edu

†e-mail: colgate@northwestern.edu

‡e-mail: peshkin@northwestern.edu
}

Watanabe and Fukui [9] developed the first ultrasonic vibrating plate capable of controlling the surface roughness displayed to a bare finger using the squeeze film effect. A squeeze film is high pressure air that forms between two relatively flat surfaces when one is vibrated at high frequency. If the high pressure overcomes the normal force, then one of the objects will float on a "cushion" of air. The $76 \mathrm{kHz}$ vertical motion in Watanabe's device created a squeeze film of air that was shown to mask the roughness of fine-grit sand paper. Biet et al. [1] used an array of piezoelectric actuators glued to the underside of a metallic sheet. The actuators set the sheet into an ultrasonic resonance mode. The vertical vibrations have an amplitude on the order of $1 \mu \mathrm{m}$ which creates a squeeze film of air between the bare finger and the metallic sheet. Frictional forces can be modulated by adjusting the amplitude of the vibration.

In a similar approach, the Tactile Pattern Display (TPaD) [10], is composed of a piezoelectric actuator attached to the underside of a glass plate. Its tactile interface is based on modulating the surface friction of the glass plate. There is convincing preliminary evidence that a user actively exploring the surface of the TPaD can experience the haptic illusion of textures and surface features such as roughness, fish scales, and smooth bumps.

In this paper we introduce a novel device that builds on the TPaD concept, but can actively apply forces. The TPaD can modulate friction; the ShiverPad can be used as a source of shear force for a finger, regardless of the direction of travel.

The ShiverPad creates a net force on a finger by alternating between low and high friction at the same frequency that the TPaD is oscillated laterally. During each high friction phase an impulse is provided to the finger. The time average of these impulses creates a non-zero net force. In Figure 1 the process for creating a rightward net force is explained.

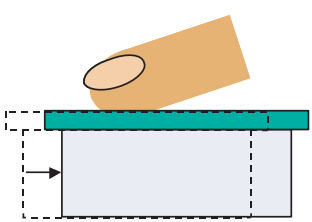

(a) Rightward movement with high friction

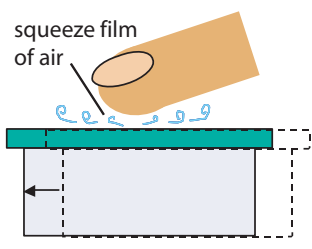

(b) Leftward movement with low friction
Figure 1: The ShiverPad generating a rightward net force. (a)The $\mathrm{TPaD}$ is moved to the right creating a rightward impulse on the finger. (b) The squeeze film is turned on and the low-friction TPaD slips back to the left to prepare for another rightward impulse.

This concept is easily extended to two dimensions by swirling a $\mathrm{TPaD}$ in small, in-plane, circles. As the TPaD swirls, its velocity vector will sometimes line up with the desired force direction. Around that time, the TPaD could be set to its high friction state and an impulse of force applied to the finger. During the remainder of the "swirl" cycle, the TPaD is set to low friction so that it negligibly effects the force on the finger. Since the velocity vector passes through all $360^{\circ}$ during the swirl, it would be possible to 
create forces in any in-plane direction.

The ShiverPad concept has the potential to evolve into a visually transparent device capable of controlling shear force on a bare finger. It would allow the user to experience compliance, viscosity, or any other force field constrained to the two dimensions of the plane, and give us increased ability to create the haptic illusion of textures and surface features. This paper discusses the design and performance of the 1DOF ShiverPad.

\section{The ShiverPad deVICE}

We have created a 1DOF device capable of applying forces to a finger in the left or right direction. The ShiverPad in Figure 2 consists of a speaker connected rigidly to a $\mathrm{TPaD}$ on a linear slider. The speaker, which serves only as a linear actuator, is sinusoidally activated at frequencies between 20 and $100 \mathrm{~Hz}$, causing the TPaD to move laterally at the same frequency.

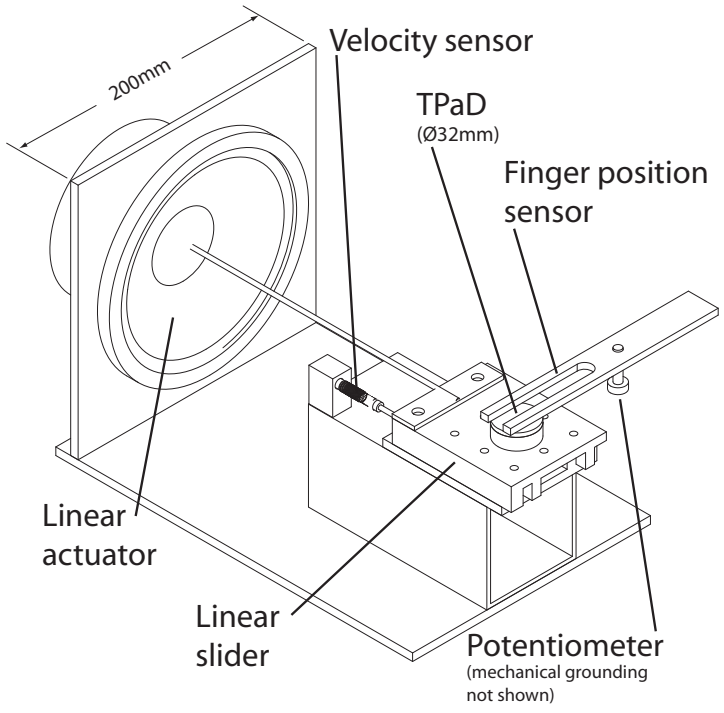

Figure 2: The ShiverPad device.

Friction is modulated on the glass surface of the TPaD by applying a $39 \mathrm{kHz}$ sinusoidal voltage to the piezoelectric element mounted on the underside of the glass. The $39 \mathrm{kHz}$ signal is generated by a AD9833 wave form generator chip and amplified to $\pm 20 \mathrm{~V}$ using an audio amplifier. When applied to the piezoelectric, it causes resonant vibrations of the glass plate. As described by Winfield et. al. [10], these vibrations produce a squeeze film of air underneath the fingertip, leading to a reduction of friction. They found that at high excitation voltages, the friction between the glass and a finger is approximately $\mu=0.15$, while at zero voltage, the surface has the friction of normal glass (approximately $\mu=0.95$ ).

A programable integrated circuit (PIC) generates the low frequency signal for the speaker and issues the command to the wave form generator to start/stop the $39 \mathrm{kHz}$ signal. Since it provides both functions, it can dictate the phase relationship between the TPaD's friction level and the lateral motion.

To measure the velocity of the TPaD, we have fabricated a device consisting of a stationary coil of wire penetrated by a magnet that oscillates laterally with the $\mathrm{TPaD}$. Velocity is proportional to output voltage, and position is found using numerical integration. This device was calibrated at multiple frequencies using a MEMS $2 \mathrm{~g}$ accelerometer (LIS3L06AL).

To measure finger position, a potentiometer is attached to a forkshaped piece of plastic that surrounds the finger and moves with it. As the finger moves, the voltage output of the potentiometer is passed to the control algorithm in the PIC chip. The force applied to the finger can be altered in real-time based on the potentiometer position.

\section{METHOD OF FORCE MEASUREMENT}

Measuring the forces on a real human finger would require mounting force sensing equipment on the oscillating slider. Our device is not built with this capability, so to measure lateral forces, we used a proxy finger pad attached to a $1 \mathrm{DOF}$ tension/compression load cell ( \pm 250 g capacity)

\subsection{The proxy finger}

Effort was made to find an object that approximates the properties of a human finger. We tried various rubber pads, but found them too stiff - the experimental data did not match the subjective feel. We tried organic substitutes like grapes and cherries because they have similar shape, density, and compliance to the human finger. The grapes worked well but they often leached water through their skin. To prevent this from occurring, electrical tape was stretched around the contact area of the grape.

It was found that the electrical tape to glass interface sometimes displayed significant stiction. To remove this effect, the smooth electrical tape surface was sandpapered. The experiments in this paper use a grape wrapped in sandpapered electrical tape as the proxy finger pad.

The grape "fingertip" is secured to an aluminum "finger" with electrical tape, and the aluminum finger is threaded onto the load cell. There is some compliance in the fingertip-to-finger connection, but since there is similar compliance in the human finger, we find it appropriate.

\subsection{Setting normal force}

The load cell is allowed to move vertically on a linear slider. The weight of the load cell and proxy finger is suspended by a lowstiffness spring. Vertical position of the finger is adjustable via a thumb nut. After the finger is lowered to within close proximity $(<0.5 \mathrm{~mm})$ of the $\mathrm{TPaD}$, the normal force is controlled by adding weight to the finger. A normal load of $392 \mathrm{mN}(40 \mathrm{~g})$ was used for most experiments in this paper. Figure 3 shows the substitute finger, the load cell, and the fixture used for controlling its position.

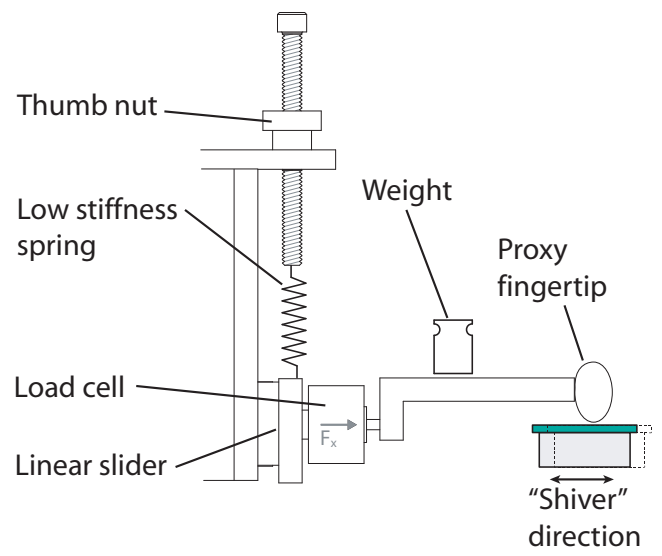

Figure 3: The force measurement device.

\section{Characterization OF FORCE GENERATION}

Forces are created by alternating between low and high friction at the same frequency that the TPaD is being oscillated laterally. To produce a net leftward force, the TPaD is set to high friction while 
its velocity is leftward and set to low friction when its velocity is rightward. The $\mathrm{TPaD}$ alternates between pushing the finger to the left and slipping underneath the finger back to the right. This "pushslip" cycle repeats itself, and the series of strong leftward impulses followed by weak rightward impulses results in a net force to the left. These impulses can be seen in the unfiltered force signal in Figure 5.

\subsection{The effect of phasing on force}

By changing the phase angle between the lateral velocity and the $\mathrm{TPaD}$ on/off signal, we are able to change the direction and magnitude of the net force. Here we define $\Phi_{o n}$ as the phase angle of the lateral velocity when the TPaD turns on. This concept is shown graphically in Figure 4. In all of the data presented in this paper, the $\mathrm{TPaD}$ is in the on state for half $\left(180^{\circ}\right)$ of the full cycle.

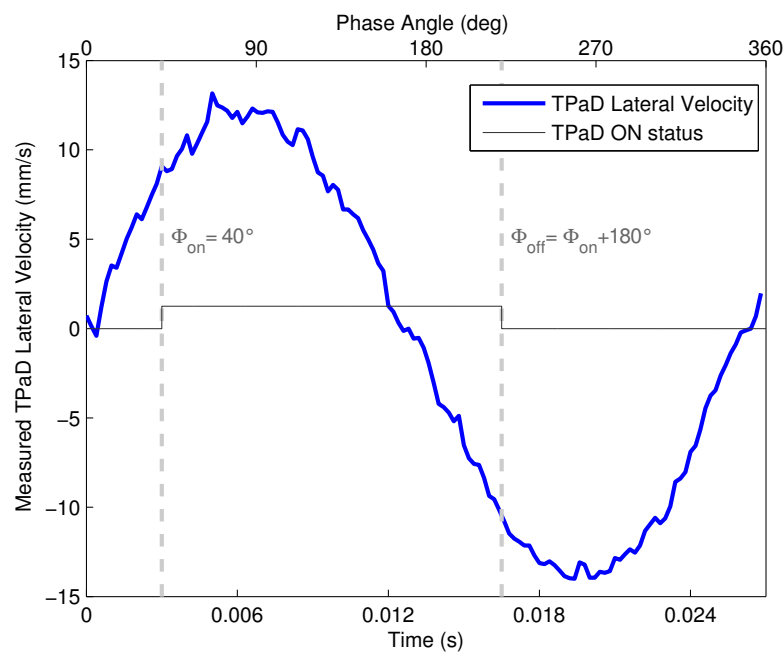

Figure 4: $\Phi_{o n}$ is defined as the phase angle of lateral velocity when the TPaD turns on. In this example, the TPaD turns on at $\Phi_{\text {on }}=40^{\circ}$ and remains in the on state for $180^{\circ}$.

We hypothesize that maximum force will be produced if the squeeze film is present when the velocity is in one direction and not present when the velocity is in the other direction. So with no delays in the system, we would expect that turning the TPaD on at $0^{\circ}$ would produce the largest leftward force and $\Phi_{\text {on }}=180^{\circ}$ would produce the greatest rightward force.

To determine which phasing creates the largest magnitude force, $\Phi_{\text {on }}$ was rotated slowly from 0 to $360^{\circ}$ over the course of about 2 seconds. To find the net force, the unfiltered force data was passed through a second-order, lowpass, butterworth, zero-phase filter $\left(f_{\text {cutoff }}=10 \mathrm{~Hz}\right)$. The filtered force signal is shown in Figure 5. The circled maximum force points correspond to the two "optimum $\Phi_{\text {on }}$ " values for this particular frequency and amplitude of lateral oscillation. This maximum net force in the $100 \mathrm{mN}$ range is easily perceivable to the authors and the literature shows that this magnitude is generally perceivable to humans [6] [2] [10].

Figure 5 shows that the net force changes as $\Phi_{\text {on }}$ is rotated over time. The $\Phi_{\text {on }}$ value in any given velocity cycle was found by comparing the TPaD status signal to the velocity signal (as shown in Figure 4). That data was then plotted against the filtered force data in Figure 5. The result in Figure 6 provides more specific information about the values of $\Phi_{\text {on }}$ that optimize force. Although the exact relationship between force and $\Phi_{\text {on }}$ is dependent on oscillation amplitude and frequency, this data is representative of a wide range of amplitudes that produce forces noticeable to a human.

The data shows that the optimum leftward force is produced at $\Phi_{o n}=340^{\circ}$, and the optimum rightward force is produced at $\Phi_{o n}=$

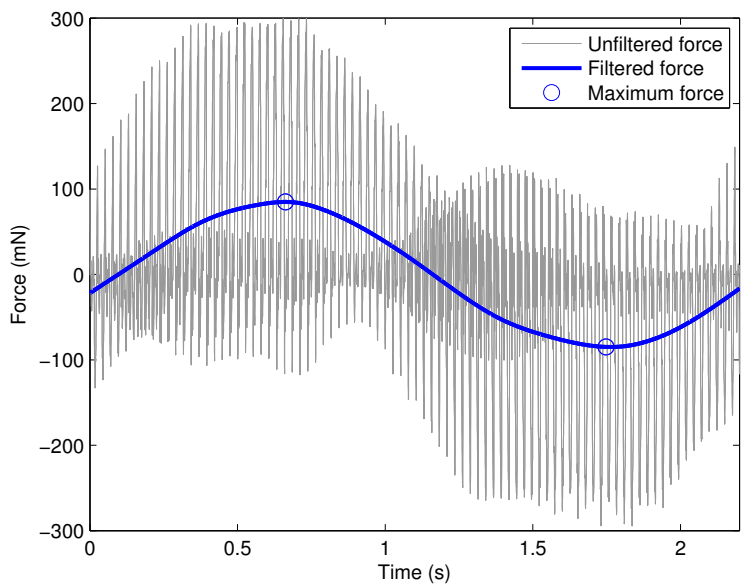

Figure 5: The unfiltered $40 \mathrm{~Hz}$ force signal has the same frequency as the lateral motion of the TPaD. $\Phi_{\text {on }}$ is rotated through all phase angles at $0.5 \mathrm{~Hz}$ and the net (filtered) force changes accordingly. The circled maximum force points occur at the "optimum $\Phi_{\text {on }}$ " values.

$160^{\circ}$. We speculate that the need for this $20^{\circ}$ phase advance is due to the time required to create and decay the squeeze film. Also note that zero net force is expected at $\Phi_{\text {on }}=270^{\circ}$ but occurs at $\Phi_{\text {on }}=250^{\circ}$.

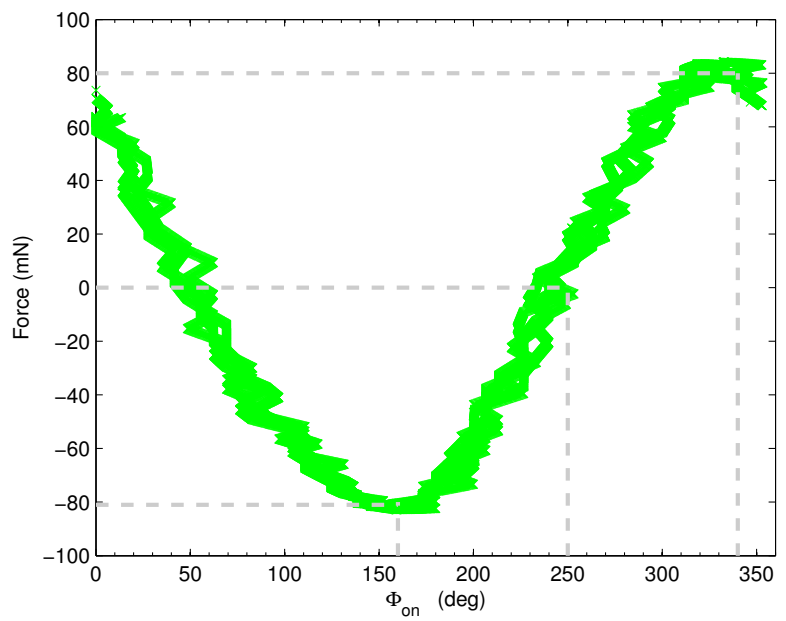

Figure 6: As $\Phi_{\text {on }}$ is changed, the net force shifts from leftward to rightward and back again. The optimum $\Phi_{\text {on }}$ values that produce the maximum leftward and rightward forces are marked. Additionally, one of the two $\Phi_{\text {on }}$ values that produces zero net force is marked.

\subsection{The effect of oscillation amplitude on force}

It was found experimentally that as the amplitude of lateral displacement increases, the average net force increases proportionally at first and then reaches a maximum. Figure 7 shows this trend for various lateral oscillation frequencies. Each data point represents the net force produced when $\Phi_{o n}$ is optimized for that particular amplitude and frequency.

The asymptotic behavior in Figure 7 is due to the nature of coulomb friction. Once amplitudes are high enough to keep the finger and $\mathrm{TPaD}$ predominately in sliding contact, the finger will experience a force of $\mu_{\text {glass }} F_{N}$ when the velocity is in one direction and $-\mu_{o n} F_{N}$ when velocity is in the other direction. $\mu_{\text {glass }}$ is the 


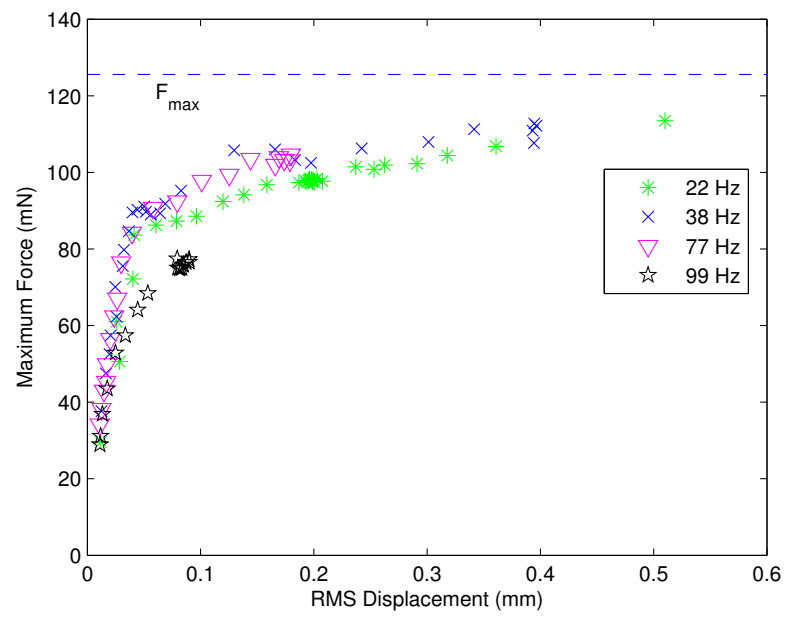

Figure 7: Each data point represents the net force produced when $\Phi_{o n}$ is optimized for that particular amplitude and frequency of oscillation. Parameters of interest: $F_{N}=392 \mathrm{mN}, \mu_{\text {glass }}=0.70, \mu_{o n}=0.06$.

kinetic coefficient of friction of the glass; $\mu_{o n}$ is the kinetic coefficient of friction of the TPaD when it is in its lowest friction state; and $F_{N}$ is the normal force.

To find the theoretical maximum net force that the ShiverPad can create, we assume that the finger experiences each of the two force levels for half of the total cycle. The time-averaged force is then just the simple average of the two force levels. Therefore, the equation for the maximum net force, $F_{\max }$, is

$$
F_{\max }=\frac{\left(\mu_{\text {glass }}-\mu_{o n}\right) F_{N}}{2}
$$

The value of the asymptote line in Figure 7 is calculated using Eqn 1 , where $\mu_{\text {glass }}=0.70$ was found by recording the maximum force while sweeping the proxy finger across the surface while the $\mathrm{TPaD}$ and lateral oscillator were quiescent; $\mu_{o n}=0.06$ was found similarly but with the TPaD turned on; and $F_{N}=392 \mathrm{mN}$ was from a $40 \mathrm{~g}$ weight.

\subsection{Frequency selection}

It is important to note here that since the force is applied in impulses at frequencies between 20 and $100 \mathrm{~Hz}$, the user is aware of not only the overall force in one direction, but also the undesirable underlying vibration of the $\mathrm{TPaD}$. At $20 \mathrm{~Hz}$, the underlying lateral vibration of the TPaD is very apparent to the user. At higher frequencies (e.g. $120 \mathrm{~Hz}$ ) the net force on the finger is significantly reduced. As a compromise, $40 \mathrm{~Hz}$ was used for most experiments.

We speculate two possible causes for the reduction in force as frequency increases:

1. As seen in Figure 7, small amplitude displacements cannot create significant forces. At high frequency, our device is not capable of achieving high amplitude displacement.

2. At high frequency the squeeze film must form and dissipate quickly. It is possible that the squeeze film does not have time to completely dissipate prior to being re-initiated, so that maximum and minimum $\mu$ values are never achieved.

As a side note, one design method for reducing the finger's exposure to the lateral vibration is to keep the TPaD continually turned on until force production is needed. In this strategy the squeeze film isolates the user from the underlying low frequency vibration making it almost unnoticeable until force is applied.

\subsection{The effect of finger exploration velocity}

When the amplitude of oscillation is large enough to bring the forces near the $F_{\max }$, increasing amplitude further provides negligible increase to the force on a stationary finger. On the other hand, if the user is actively exploring the surface, his finger velocity could cause the relative velocity between the finger and plate to become small, reducing the net force. Therefore, the higher the finger exploration velocities, the higher the oscillation amplitude required to maintain the target force.

An idea for acceptable finger exploration velocities can be gained by plotting the same data in Figure 7 against velocity instead of displacement. In Figure 8 we see that a TPaD oscillating at $77 \mathrm{~Hz}$ reaches about $80 \%$ of force production around $20 \mathrm{~mm} / \mathrm{s}$ RMS velocity. We see that force produced by $77 \mathrm{~Hz}$ oscillation is very sensitive to changes in RMS velocity below $20 \mathrm{~mm} / \mathrm{s}$, but insensitive above $20 \mathrm{~mm} / \mathrm{s}$. Therefore, we can predict that the forces produced by a ShiverPad oscillating at $20 \mathrm{~mm} / \mathrm{s}$ are susceptible to finger velocity changes, but a ShiverPad running at $60 \mathrm{~mm} / \mathrm{s}$ will allow the finger to travel at speeds up to about $40 \mathrm{~mm} / \mathrm{s}$ before a significant reduction in force occurs.

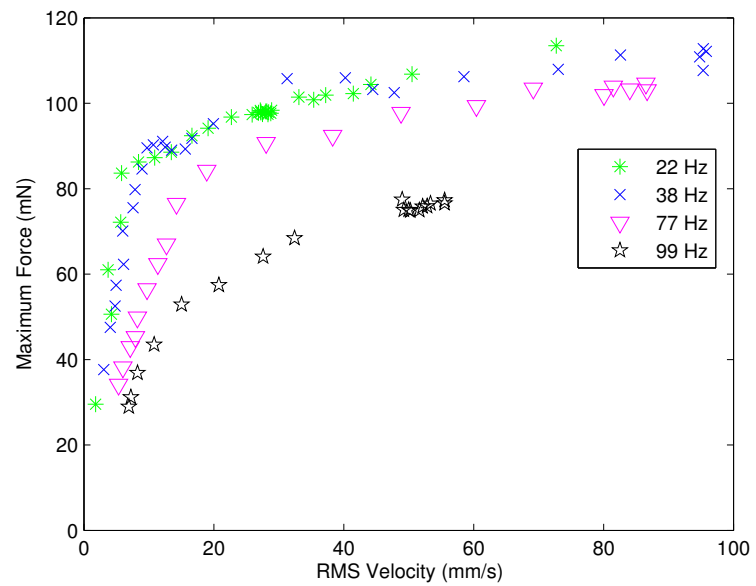

Figure 8: The data in Figure 7 replotted as a function of TPaD RMS velocity.

\section{DisPlaying FORCE FIELDS}

Since the ShiverPad is effectively a source of force, it is possible to display any arbitrary force field. We could chose to display a spring, damper, or other primitive, but here we have chosen to display line sinks and sources. At any given moment in time, the device has a constant force field across its surface, so to create the perception of a non-constant force field, it is necessary to change force with finger position. In practice, as the finger moves across the surface, $\Phi_{o n}$ is adjusted to produce the force of desired direction and magnitude. In Figure 9 we provide a top-view of what a line-source force field looks like, and the $\Phi_{\text {on }}$ command we use to generate such a field.

The commanded $\Phi_{\text {on }}$ in Figure 9 is derived from the Force versus $\Phi_{\text {on }}$ relationship in Figure 6. To create a line source we want zero force along the centerline, so we have chosen $\Phi_{\text {on }}=250^{\circ}$ at $x=0$. On the right and left edges of the vector field where maximum force is required, the $\Phi_{\text {on }}$ command takes on the optimum $\Phi_{\text {on }}$ angles from Figure $6\left(340^{\circ}\right.$ and $\left.160^{\circ}\right)$

\subsection{Method of force measurement}

To measure force on the finger in these force fields, it is necessary to move the proxy fingertip over the surface of the TPaD. To accomplish this, the entire force measuring fixture in Figure 3 was 

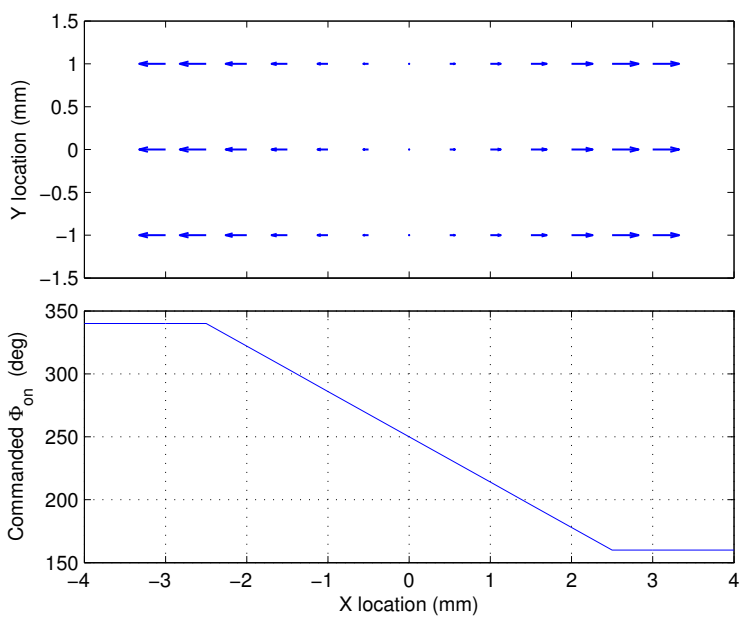

Figure 9: A line-source force field and the $\Phi_{\text {on }}$ request the ShiverPad uses to generate it.

slowly shifted by hand. To find fingertip position, the proxy fingertip was placed between the tongs of the finger positioning fork. The force created by friction in the finger position potentiometer was measured and found to be negligible. Normal force was set as described in Section 3.2 except that the tongs of the fork were placed around the fingertip prior to being lowered into close proximity to the $\mathrm{TPaD}$.

\subsection{Results and discussion}

Figure 10 shows the data from four different force fields. There are two line-sources and two line-sinks, each of which has a "stiff" and "compliant" version. The raw data has been provided in the force vs. position format, but to provide a more intuitive idea for the tactile experience, we have also integrated the data to form the "potential function". The potential function is defined as $V(x)=\int F(x) d x$, where $F(x)$ is the force on the finger as a function of position, $x$. The results from Robles-De-La-Torre and Hayward [6] suggest that the shape of the potential function is similar to the perceived shape of a virtual bump or hole.

When viewing the data from the perspective of the potential function, instead of seeing a stiff planar line-source, we see a steep bump in the surface. Similarly, the compliant planar line-sink can be thought of as a shallow hole in the surface.

\section{CONCLUSION}

The ShiverPad is capable of applying and controlling the net shear force on a finger. As with any controllable force source, it allows us to display force fields of our choosing when coupled with finger position feedback. We have shown the capability of displaying line sources and sinks and reminded the reader of the idea that they can be viewed as planar force fields, or 3D protrusions and depressions.

The current limitations to this capability are

- The underling vibration is evident to the user.

- Friction coefficient and normal force limit the maximum force output.

- Our device is limited to low finger exploration speeds (this can be remedied by using higher lateral TPaD velocities).

- Our device is limited to $1 \mathrm{DOF}$ (the concept could be easily extended to a $2 \mathrm{DOF}$ version).
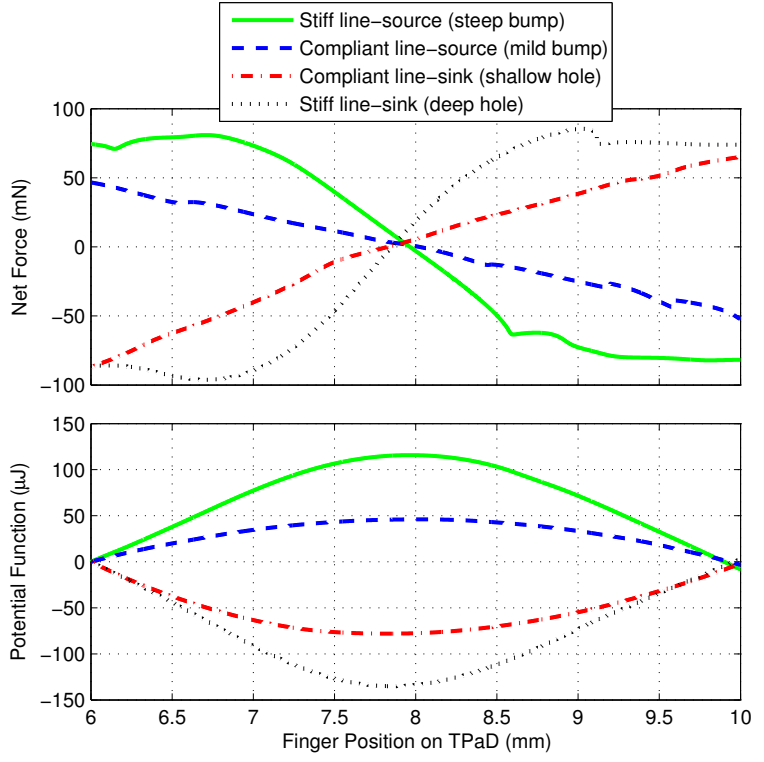

Figure 10: Two line-sources and two line-sinks. The same data displayed as a position-dependent force and a potential function

While our prototype has limitations, it is a first step towards a visually transparent tactile display capable of applying any arbitrary shear force to a finger. It would have the capability of displaying a 2D world composed of springs, dampers, masses, and other forces, but also (and maybe more importantly), by using the idea that lateral force can create the illusion of shape, it could produce the illusion of $3 \mathrm{D}$ textures and shape on its $2 \mathrm{D}$ surface.

Future work includes

- Increasing the lateral oscillation frequency in an effort to make it is less perceptible to the user.

- Developing a predictive model of the force production mechanism.

- Using the ShiverPad to produce illusions of textures and surface features not possible with a passive TPaD.

\section{ACKNOWLEDGEMENTS}

The authors would like to acknowledge the National Science Foundation grant 0413204 for support of this work.

\section{RefERENCES}

[1] M. Biet, F. Giraud, and B. Lemaire-Semail. Implementation of tactile feedback by modifying the perceived friction. European Physical Journal Appl. Phys., 43:123135, 2008.

[2] S. M. Biggs, S. Haptic Interfaces, chapter 5, pages 93-115. Published by Lawrence Erlbaum Associates, 2002.

[3] M. Minsky. Computational Haptics: The Sandpaper System for Synthesizing texture for a force-feedback display. PhD thesis, Massachusettes Institute of Technology, Cambridge, MA, 1995.

[4] J. Pasquero and V. Hayward. Stress: A practical tactile display with one millimeter spatial resolution and $700 \mathrm{hz}$ refresh rate. Dublin, Ireland, July 2003.

[5] G. Robles-De-La-Torre. Comparing the Role of Lateral Force During Active and Passive Touch: Lateral Force and its Correlates are Inherently Ambiguous Cues for Shape Perception under Passive Touch Conditions. pages 159-164, 2002.

[6] G. Robles-De-La-Torre and V. Hayward. Force can overcome object geometry in the perception of shape through active touch. Nature, 412:445-448, July 2001. 
[7] M. Takasaki, H. Kotani, T. Mizuno, and T. Nara. Transparent surface acoustic wave tactile display. Intelligent Robots and Systems, 2005. (IROS 2005). 2005 IEEE/RSJ International Conference on, pages 3354-3359, Aug. 2005.

[8] V. Vincent Levesque and V. Hayward. Experimental evidence of lateral skin strain during tactile exploration. In Proc. of Eurohaptics, Dublin, Ireland, July 2003.

[9] T. Watanabe and S. Fukui. A method for controlling tactile sensation of surface roughness using ultrasonic vibration. Robotics and $\mathrm{Au}$ tomation, 1995. Proceedings., 1995 IEEE International Conference on, 1:1134-1139 vol.1, May 1995.

[10] L. Winfield, J. Glassmire, J. E. Colgate, and M. Peshkin. T-pad: Tactile pattern display through variable friction reduction. World Haptics Conference, pages 421-426, 2007.

[11] A. Yamamoto, T. Ishii, and T. Higuchi. Electrostatic tactile display for presenting surface roughness sensation. pages 680-684, December 2003 